\title{
Effects of index-mismatch-induced spherical aberration in pump-probe microscopic image formation
}

\author{
Peter T. Fwu, Po-Hsiang Wang, Chi-Kuan Tung, and Chen-Yuan Dong
}

\begin{abstract}
Pump-probe fluorescence microscopy has been demonstrated to be a powerful tool for obtaining three-dimensional, time-resolved information in bioimaging applications. However, the use of this technique can be complicated by the fact that the different wavelengths used to achieve pump-probe microscopy can result in wavelength-dependent spherical aberration, thus limiting the usefulness of the technique. We address this issue by investigating the effects of refractive-index-mismatch-induced spherical aberration on pump-probe image formation. We model the effects by considering pumpprobe imaging performed with an objective with a numerical aperture of 0.75 focusing through an oil-water interface. Our results show that spherical aberration has the greatest effect in degrading an axial point-spread function. In addition to signal loss, the redistribution of signal strength along the axial direction results in broadening of the FWHM of the plane response function. The inclusion of confocal detection tends to improve image resolution but at a significant loss of signal strength. (C) 2005 Optical Society of America
\end{abstract}

OCIS codes: $180.0180,190.4180$.

\section{Introduction}

In recent years, microscopic imaging techniques capable of acquiring high-contrast, three-dimensional images in biological specimens have attracted considerable attention. Whereas confocal microscopy has proved to be a powerful technique for the characterization of biological specimens, the capabilities of scanning optical microscopy were further advanced by the development of other imaging modalities. Two-photon fluorescence microscopy, pioneered by Webb and co-workers at Cornell University, 1,2 has become extremely useful in many areas of microscopic imaging. The ability of multiphoton microscopy to limit the excitation volume to the focus is significant in that confocallike, high-contrast images can be generated without the use of detection pinholes. The limited sample photodamage to the focus also promotes longevity of biological specimens. Finally, near-infrared excita-

The authors are with the Department of Physics, National Taiwan University, Taipei 106, Taiwan. C.-Y. Dong's e-mail address is cydong@phys.ntu.edu.tw.

Received 28 October 2004; revised manuscript received 4 January 2005; accepted 5 January 2005.

0003-6935/05/204220-08\$15.00/0

(C) 2005 Optical Society of America tion photons are absorbed and scattered less by many biological specimens and can be further focused into the samples, thus permitting threedimensional imaging without the need for histological procedures. ${ }^{2}$

More recently, a time-resolved imaging technique based on the pump-probe excitation of specimen fluorophores was developed, and it shares some of the advantages of multiphoton techniques. The way in which this method works is shown in Fig. 1. In this approach, two laser sources with different repetition frequencies are focused and overlapped at the fluorescent sample. In one implementation, the wavelength of one laser (the pump) is chosen to excite the sample while the wavelength of the second laser (the probe) is chosen to induce stimulated emission from the flurophores in the excited state. As the two lasers have different repetition frequencies, the combined effects of excitation and stimulated emission result in modulation of excited-state population at the crosscorrelation frequency harmonics. In addition, because the pump-probe effect is effectively generated in regions where the two beams overlap, the crosscorrelation signal is dependent on the spatial integral of the product of the two laser intensity profiles. In short, pump-probe, cross-correlation (c.c.) signal $F_{c c}(t)$ generated from a species of fluorescent molecules with lifetime $\tau$ is given by 


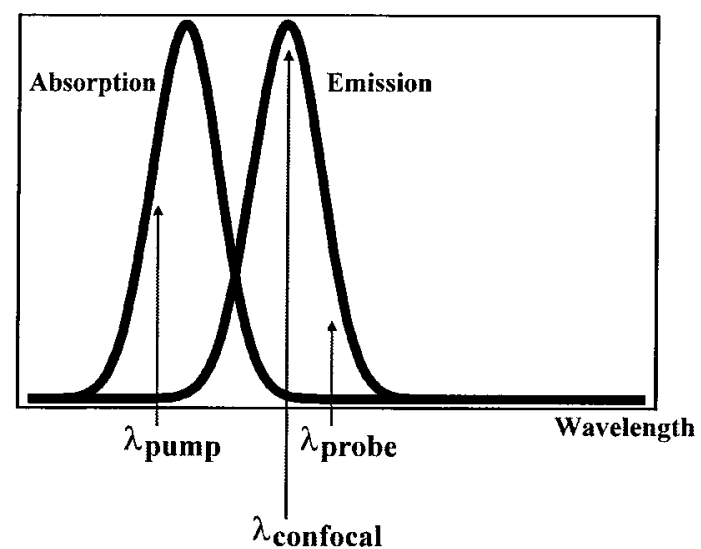

Fig. 1. Pump-probe fluorescence microscopy.

$F_{c c}(t) \sim \frac{1}{\left(1+\omega^{2} \tau_{m}^{2}\right)^{1 / 2}} \cos \left[\left(\omega^{\prime}-\omega\right) t-\phi\right] \int I(\mathbf{r}) I^{\prime}(\mathbf{r}) \mathrm{d}^{3} r$,

where $\omega$ and $I(\mathbf{r})$, respectively, denote the modulation frequency and the spatial intensity distribution of the pump beam. The corresponding quantities of the probe beam are represented by primed symbols. In addition, $m=1 /\left(1+\omega^{2} \tau_{m}^{2}\right)^{1 / 2}$ and $\phi=\tan ^{-1}\left(\omega \tau_{\phi}\right)$, respectively, represent the modulation and the phase of the cross-correlation signal that can be used for determination of specimen lifetime. The subscript of lifetime $\tau$ designates that determined from the modulation and the phase of the cross-correlation signal. Note that it is the dependence of the cross-correlation signal on the spatial integral of the product of pump and probe beam intensities $\int I(\mathbf{r}) I^{\prime}(\mathbf{r}) \mathrm{d}^{3} r$ that provides the spatial specificity of this technique. Pumpprobe fluorescence microscopy implemented in this way has been demonstrated in bioimaging applications, and the imaging resolution has also been investigated. Although pump-probe microscopy is similar to confocal or multiphoton microscopy in its ability to generate optical sectional images, it is unique in one respect. As relation (1) shows, the modulation and the phase of the cross-correlation signal at frequency $\left|\omega^{\prime}-\omega\right|$ can be analyzed for highfrequency lifetime information at a pump modulation frequency of $\omega$. Therefore high-frequency information can be acquired from a specimen without fast optical detectors. It has been demonstrated that the pumpprobe methodology may be conveniently used to measure sample harmonic content into the gigahertz range with a standard photomultiplier tube that responds in the hundreds-of-megahertz range. ${ }^{3}$ In the stimulatedemission approach it has been shown that one can achieve polarization imaging by altering the polarization of the pump and the probe beams. ${ }^{4}$ In addition, intensity-modulated laser diodes can also be used to generate the pump-probe effect for microscopic imaging. ${ }^{5}$ Furthermore, although we have limited our discussion to the stimulated-emission approach, the pump-probe technique can be applied if the wave-

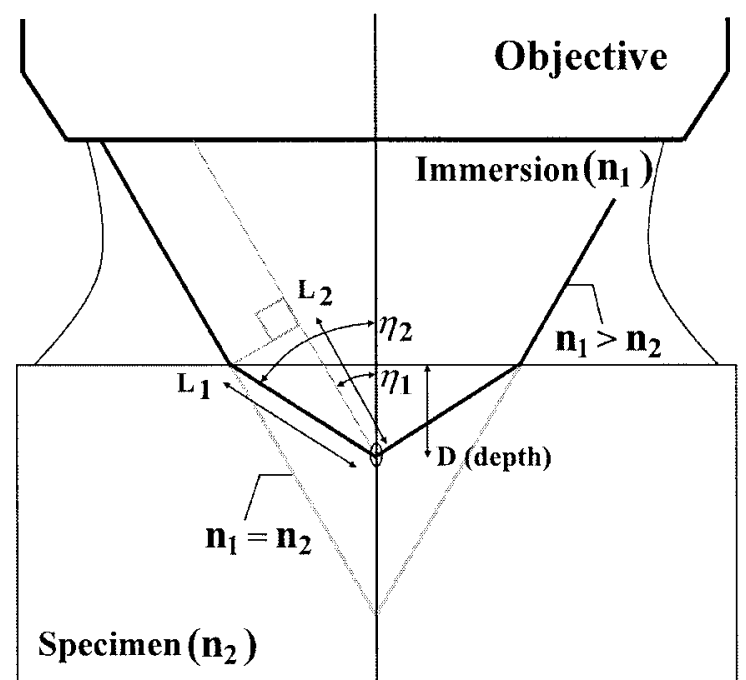

Fig. 2. Index-mismatch-induced spherical aberration in microscopic image formation.

lengths of both the pump and the probe beams are chosen for sample excitation. The transient-absorption approach can also modulate the excited-state population at the low, cross-correlation frequency; the resultant cross-correlation fluorescence signal will also contain lifetime information on the excited-state molecules. 6,7

\section{Spherical Aberration and Microscopic Image Formation}

In bioimaging applications, specimen optical properties such as the variation of refractive indices and scattering coefficients can lead to a degradation of image quality. As shown in Fig. 2, for mismatched refractive indices the refraction that occurs at the interface of an oil-water boundary leads to a change in the directions of the propagating light rays. The result of such deviation is the formation of a new focal position and the generation of an aberrated pointspread function (PSF), given by

$$
I(u, v, D)=\left|\int_{0}^{1} P(\rho, D) \exp \left(i u \rho^{2} / 2\right) J_{0}(v \rho) \rho \mathrm{d} \rho\right|^{2},
$$

where $P(\rho, D)=\exp [i \Phi(\rho, D)]$ is the pupil function that accounts for the phase difference of light rays $L_{1}$ and $L_{2}$, given by $\Phi(\rho, D)=(2 \pi D / \lambda) n_{1} \sin \delta_{1}\left[\left(\csc ^{2} \delta_{2}\right.\right.$ $\left.\left.-\rho^{2}\right)^{1 / 2}-\left(\csc ^{2} \delta_{1}-\rho^{2}\right)^{1 / 2}\right]$. Distance $D$ represents the distance from the interface to the altered focal point, $\delta_{1}$ is the half-angle of the objective, and $\delta_{2}$ is determined from the relationship $n_{1} \sin \delta_{1}$ $=n_{2} \sin \delta_{2}$. The formalism described by Eq. (2) has been widely applied to a number of refractive-indexmismatch problems. ${ }^{8-10}$ And this is the formalism that we adopt here with which to investigate the effects of spherical aberration in pump-probe microscopic image formation. 

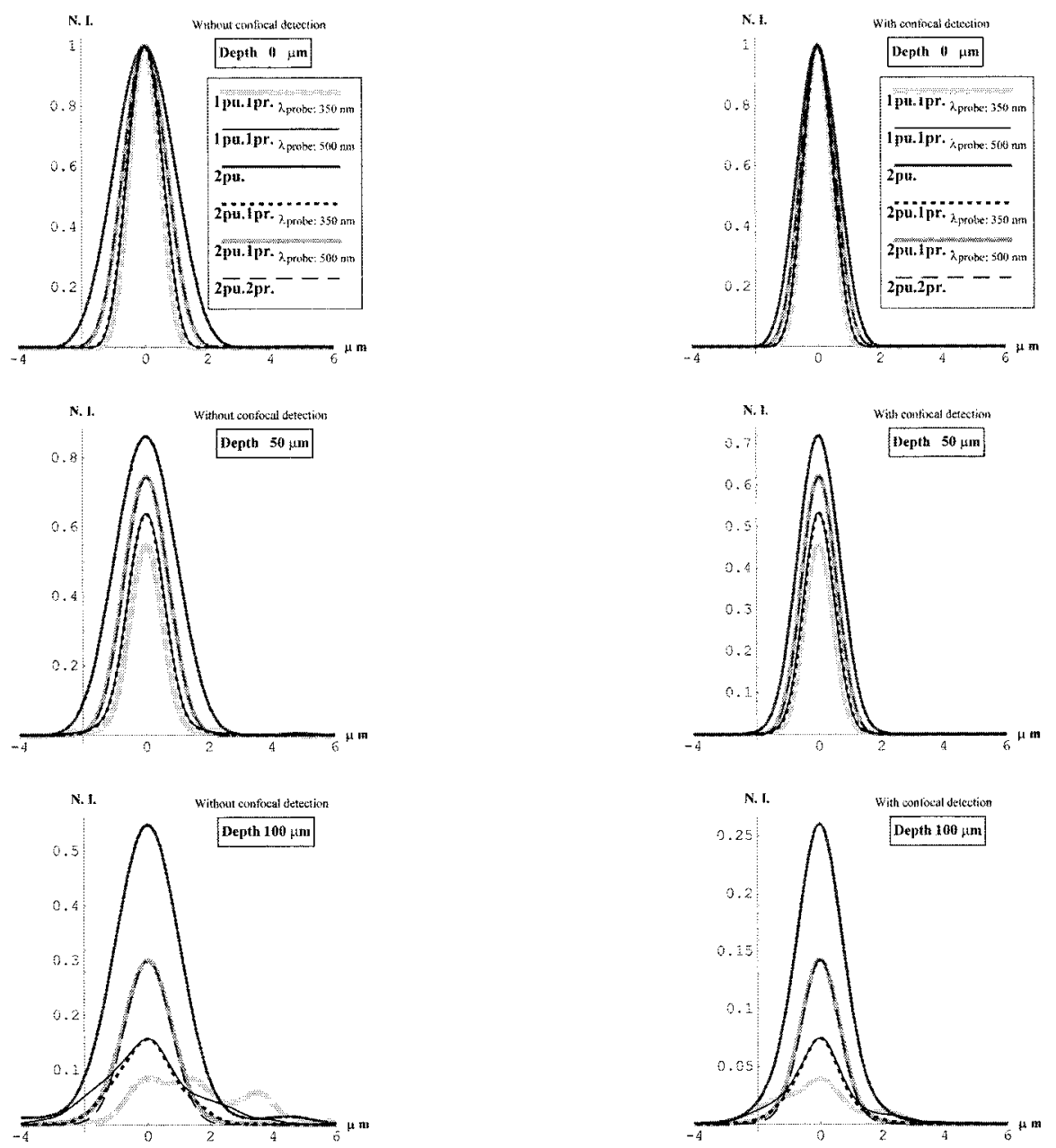

Fig. 3. Axial PSFs of pump-probe and two-photon microscopy under spherical aberration.

\section{Refractive-Index-Mismatch-Induced Spherical Aberration in Pump-Probe Microscopy}

Compared with studies of confocal and multiphoton microscopic image formation, the application of Eq. (2) for investigating spherically aberrated pumpprobe PSF involves additional degrees of complexity. As Fig. 1 shows, in typical one-photon pump and one-photon probe processes in which the excitation and the stimulated emission involve molecular interaction with one photon, three wavelengths need to be considered. The pump, the probe, and the detection wavelengths can all be different. To complicate the matter, because typical fluorescence excitation and emission are broad spectrally $(\sim 100 \mathrm{~nm})$, the possible combinations of pump, probe, and detection wavelengths can be numerous. The combination of laser wavelengths is further increased if either or both of the two lasers involved interact with the molecules by multiphoton activity. Finally, the choice of wavelengths that can be used in pump-probe microscopy is complicated by the fact that different wavelengths need to be chosen for fluorescent molecules with probe-specific spectral characteristics. Therefore it is not a trivial task to derive a generalized formalism to describe the effects of index-mismatch-induced spherical aberration in pump-probe microscopy.

Here we tackle this problem by limiting the possible spectral combinations, which we do by investigating the spherical aberration effects of one commonly used fluorphore. The fluorescent species that we chose to investigate is the nucleic acid labeled $4^{\prime}, 6$ diamidino-2-phenylindole dihydrochloride (DAPI; D-1306, excitation/emission: 358/461, Molecular Probes, Eugene, Oregon). Furthermore, we fixed the laser wavelengths that we used to excite or stimulate emission. For one-photon excitation, the pump laser was fixed at $350 \mathrm{~nm}$. The one-photon probe wavelength was chosen to be $500 \mathrm{~nm}$ (stimulated emission) or $350 \mathrm{~nm}$ (transient absorption). Furthermore, if the pump, the probe, (transient absorption), or both lasers involved two-photon operation a common wavelength of $700 \mathrm{~nm}$ was used. Note that, when two-photon operation is used for the probe beam, it is meant to induce further excitation of the groundstate molecules. In this manner the excited-state molecules can still be modulated at the cross-correlation 

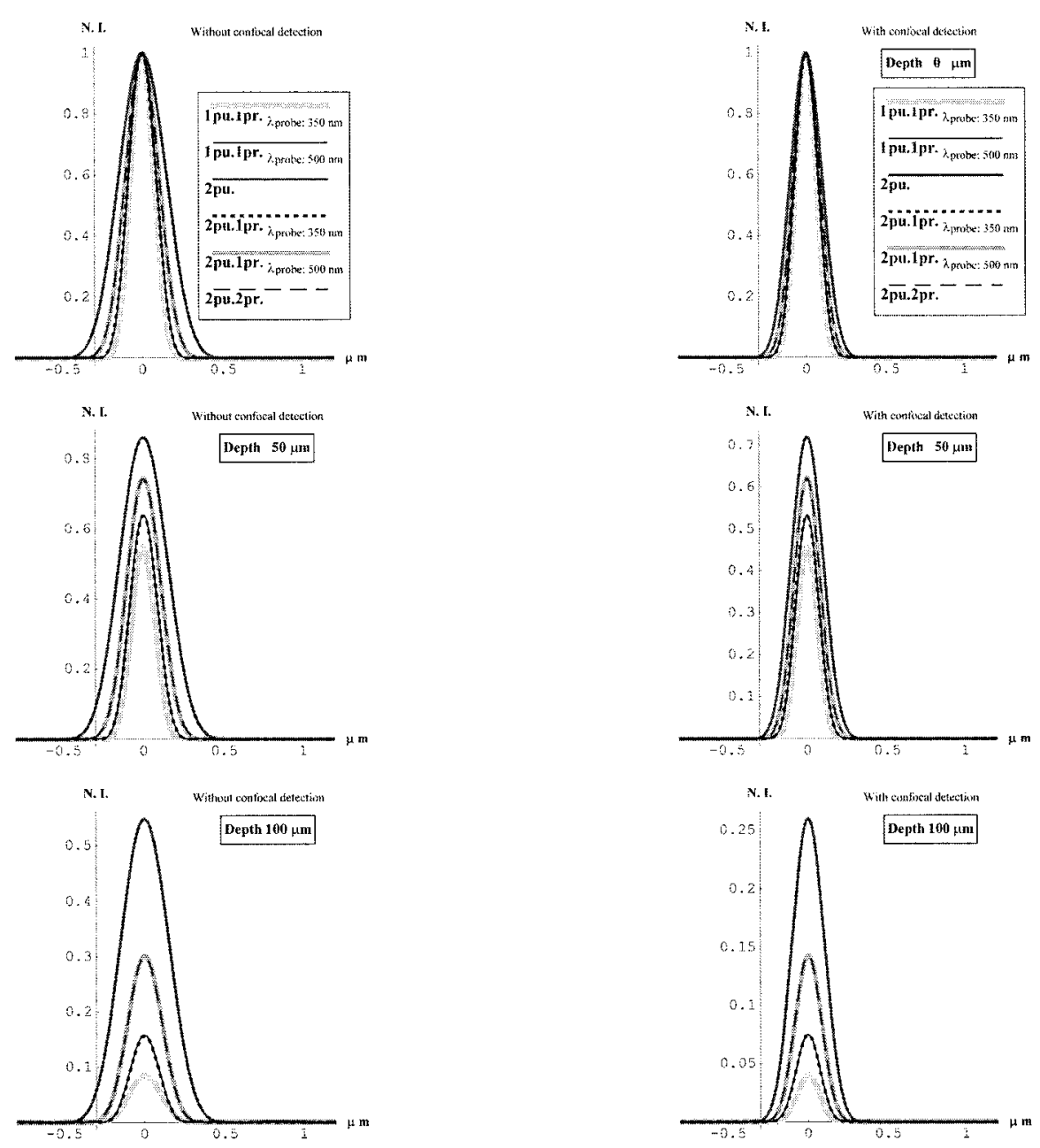

Fig. 4. Radial PSFs of pump-probe and two-photon microscopy under spherical aberration.

frequency, and one can use its phase and modulation to determine the lifetime of the specimen under study. Finally, regardless of the modes of pumpprobe interactions, the confocal detection is always performed at $450 \mathrm{~nm}$, which is near the emission peak of DAPI.

The reason for choosing a blue fluorescence species for our study is the relatively large phase difference that it introduces. To be specific, the relative phase difference among pump, probe, and detection wavelengths is larger for a blue fluorescence molecule than for a red fluorescence molecule. For example, for DAPI, the ratios of one-photon probe and detection wavelengths to that for a one-photon pump process are 1.29 and 1.43 , respectively. These ratios are expected to decrease for longer-wavelength flurophores. The reason for this phenomenon is that, regardless of the colors of the fluorescent species, the spectra of one-photon excitation and emission are $\sim 100 \mathrm{~nm}$ apart. As Fig. 2 shows, the relatively large difference among pump, probe, and detection wavelengths will result in a relatively larger phase difference at the focus, thus maximizing the effect of spherical aber- ration in pump-probe image formation. Finally, the interface that we chose for introducing spherical aberration is an oil-water interface, which is commonly encountered in bioimaging applications. The numerical aperture of the objective that we chose is 0.75 .

\section{Results and Discussion}

By varying the combinations of the pump, probe, and detection wavelengths, we calculated the PSF at five depths away from the oil-water interface (at 0, 50, $100,150$, and $200 \mu \mathrm{m})$. However, note that PSF $I(\mathbf{r}) I^{\prime}(\mathbf{r})$ described in relation (1) represents that of a one-photon pump-one-photon probe combination. If the pump or the probe involves a nonlinear process (such as two-photon absorption), a PSF involving nonlinear term $I(\mathbf{r})^{2}$ or $I^{\prime}(\mathbf{r})^{2}$ needs to be used instead. Furthermore, in our calculations we also obtained results by using a detection pinhole (at $450 \mathrm{~nm}$ ). Because as many as three wavelengths are involved in describing pump-probe microscopy, their effects on PSFs need to be carefully tracked.

We computed the effects of spherical aberration on 
Table 1. Focal Shifts, Relative Peak Intensities, Axial PSF FWHMs, Normalized Signal Levels, and Axial-P-FWHMs and Radial PSF FWHMs of Pump-Probe and Two-Photon Fluorescence Microscopy ${ }^{a}$

\begin{tabular}{|c|c|c|c|c|c|c|c|}
\hline $\begin{array}{c}\text { Depth } \\
(\mu \mathrm{m})\end{array}$ & $\begin{array}{c}\text { Excitation } \\
\text { Mode }\end{array}$ & $\begin{array}{l}\text { Focal Shift } \\
(\mu \mathrm{m})\end{array}$ & Maximum N.I. & $\begin{array}{l}\text { Axial-FWHM } \\
(\mathrm{PSF})(\mu \mathrm{m})\end{array}$ & $\begin{array}{l}\text { Radial-FWHM } \\
(\mathrm{PSF})(\mu \mathrm{m})\end{array}$ & $\begin{array}{l}\text { Normalized } \\
\text { Signal Level }\end{array}$ & $\begin{array}{c}\text { Axial-P-FWHM } \\
(\mu \mathrm{m})\end{array}$ \\
\hline \multicolumn{8}{|c|}{$1 \mathrm{pu}-1 \mathrm{pr} ; \lambda_{\text {pump }}, 350 \mathrm{~nm} ; \lambda_{\text {probe }}, 350 \mathrm{~nm} ; \lambda_{\text {confocal }}, 450 \mathrm{~nm}$} \\
\hline \multirow[t]{2}{*}{0} & $1 \mathrm{pu}-1 \mathrm{pr}$ & 0 & 1 & 1.129 & 0.174 & 1 & 1.193 \\
\hline & 1pu-1pr Conf & 0 & 1 & 0.992 & 0.152 & 1 & 1.004 \\
\hline \multirow[t]{2}{*}{50} & $1 \mathrm{pu}-1 \mathrm{pr}$ & 8.229 & 0.548 & 1.203 & 0.174 & 0.749 & 1.394 \\
\hline & $1 \mathrm{pu}-1 \mathrm{pr}$ Conf & 8.240 & 0.457 & 1.057 & 0.153 & 0.523 & 1.083 \\
\hline \multirow[t]{2}{*}{100} & $1 \mathrm{pu}-1 \mathrm{pr}$ & 14.902 & 0.085 & 3.175 & 0.230 & 0.488 & 3.554 \\
\hline & $1 \mathrm{pu}-1 \mathrm{pr}$ Conf & 16.313 & 0.040 & 2.408 & 0.162 & 0.137 & 2.578 \\
\hline \multirow{2}{*}{150} & $1 \mathrm{pu}-1 \mathrm{pr}$ & 23.046 & 0.066 & 1.845 & 0.207 & 0.384 & 3.150 \\
\hline & 1pu-1pr Conf & 22.935 & 0.019 & 1.615 & 0.187 & 0.075 & 1.982 \\
\hline \multirow[t]{2}{*}{200} & $1 \mathrm{pu}-1 \mathrm{pr}$ & 29.624 & 0.039 & 1.950 & 0.231 & 0.317 & 3.517 \\
\hline & 1pu-1pr Conf & 29.789 & 0.007 & 2.130 & 0.207 & 0.044 & 2.443 \\
\hline \multicolumn{8}{|c|}{$1 \mathrm{pu}-1 \mathrm{pr} ; \lambda_{\text {pump }}, 350 \mathrm{~nm} ; \lambda_{\text {probe }}, 500 \mathrm{~nm} ; \lambda_{\text {confocal }}, 450 \mathrm{~nm}$} \\
\hline \multirow[t]{2}{*}{0} & $1 \mathrm{pu}-1 \mathrm{pr}$ & 0 & 1 & 1.300 & 0.199 & 1 & 1.426 \\
\hline & 1pu-1pr Conf & 0 & 1 & 1.107 & 0.170 & 1 & 1.125 \\
\hline \multirow[t]{2}{*}{50} & $1 \mathrm{pu}-1 \mathrm{pr}$ & 8.232 & 0.640 & 1.379 & 0.200 & 0.791 & 1.572 \\
\hline & $1 \mathrm{pu}-1 \mathrm{pr}$ Conf & 8.241 & 0.534 & 1.158 & 0.170 & 0.594 & 1.196 \\
\hline \multirow[t]{2}{*}{100} & $1 \mathrm{pu}-1 \mathrm{pr}$ & 16.348 & 0.158 & 2.466 & 0.210 & 0.505 & 3.112 \\
\hline & $1 \mathrm{pu}-1 \mathrm{pr}$ Conf & 16.369 & 0.075 & 1.673 & 0.176 & 0.172 & 1.991 \\
\hline \multirow[t]{2}{*}{150} & $1 \mathrm{pu}-1 \mathrm{pr}$ & 22.910 & 0.073 & 2.688 & 0.251 & 0.390 & 3.053 \\
\hline & $1 \mathrm{pu}-1 \mathrm{pr}$ Conf & 22.801 & 0.022 & 2.031 & 0.216 & 0.087 & 2.124 \\
\hline \multirow[t]{2}{*}{200} & $1 \mathrm{pu}-1 \mathrm{pr}$ & 30.000 & 0.047 & 2.717 & 0.263 & 0.322 & 3.731 \\
\hline & $1 \mathrm{pu}-1 \mathrm{pr}$ Conf & 30.236 & 0.010 & 2.406 & 0.222 & 0.054 & 2.751 \\
\hline \multicolumn{8}{|c|}{$2 \mathrm{pu} ; \lambda_{\text {excitation }}, 700 \mathrm{~nm} ; \lambda_{\text {confocal }}, 450 \mathrm{~nm}$} \\
\hline \multirow[t]{2}{*}{0} & $2 \mathrm{pu}$ & 0 & 1 & 2.259 & 0.347 & 1 & 2.385 \\
\hline & 2pu Conf & 0 & 1 & 1.517 & 0.234 & 1 & 1.566 \\
\hline \multirow[t]{2}{*}{50} & $2 \mathrm{pu}$ & 8.247 & 0.862 & 2.288 & 0.346 & 0.922 & 2.462 \\
\hline & 2pu Conf & 8.248 & 0.720 & 1.560 & 0.233 & 0.763 & 1.619 \\
\hline \multirow[t]{2}{*}{100} & $2 \mathrm{pu}$ & 16.458 & 0.548 & 2.413 & 0.349 & 0.747 & 2.787 \\
\hline & 2pu Conf & 16.454 & 0.260 & 1.777 & 0.237 & 0.358 & 1.898 \\
\hline \multirow{2}{*}{150} & $2 \mathrm{pu}$ & 24.633 & 0.251 & 2.811 & 0.352 & 0.585 & 4.226 \\
\hline & 2pu Conf & 24.308 & 0.048 & 3.326 & 0.255 & 0.148 & 3.137 \\
\hline 200 & $2 \mathrm{pu}$ & 29.816 & 0.085 & 9.428 & 0.462 & 0.486 & 7.109 \\
\hline & 2 pu Conf & 30.588 & 0.019 & 3.821 & 0.300 & 0.093 & 3.523 \\
\hline $2 \mathrm{pu}-1 \mathrm{pr} ; \lambda$ & p $700 \mathrm{~nm} ; \lambda_{\text {probe }}$ & $350 \mathrm{~nm} ; \lambda_{\text {conft }}$ & $\mathrm{al}, 450 \mathrm{~nm}$ & & & & \\
\hline 0 & $2 \mathrm{pu}-1 \mathrm{pr}$ & 0 & 1 & 1.300 & 0.200 & 1 & 1.404 \\
\hline & $2 \mathrm{pu}-1 \mathrm{pr}$ Conf & 0 & 1 & 1.108 & 0.170 & 1 & 1.123 \\
\hline 50 & $2 \mathrm{pu}-1 \mathrm{pr}$ & 8.236 & 0.638 & 1.369 & 0.201 & 0.719 & 1.502 \\
\hline & $2 \mathrm{pu}-1 \mathrm{pr}$ Conf & 8.226 & 0.533 & 1.161 & 0.171 & 0.581 & 1.184 \\
\hline 100 & $2 \mathrm{pu}-1 \mathrm{pr}$ & 16.376 & 0.157 & 2.072 & 0.207 & 0.299 & 2.088 \\
\hline & $2 \mathrm{pu}-1 \mathrm{pr}$ Conf & 16.402 & 0.075 & 1.575 & 0.174 & 0.132 & 1.669 \\
\hline 150 & $2 \mathrm{pu}-1 \mathrm{pr}$ & 23.651 & 0.037 & 3.578 & 0.223 & 0.127 & 2.656 \\
\hline & $2 \mathrm{pu}-1 \mathrm{pr}$ Conf & 23.412 & 0.009 & 2.088 & 0.201 & 0.032 & 2.173 \\
\hline 200 & $2 \mathrm{pu}-1 \mathrm{pr}$ & 29.700 & 0.017 & 4.800 & 0.267 & 0.084 & 2.782 \\
\hline & $2 \mathrm{pu}-1 \mathrm{pr}$ Conf & 29.927 & 0.003 & 2.770 & 0.230 & 0.017 & 2.534 \\
\hline $2 \mathrm{pu}-1 \mathrm{pr} ; \lambda$ & p, $700 \mathrm{~nm} ; \lambda_{\text {probe }}$ & $500 \mathrm{~nm} ; \lambda_{\text {conft }}$ & $\mathrm{al}, 450 \mathrm{~nm}$ & & & & \\
\hline 0 & $2 \mathrm{pu}-1 \mathrm{pr}$ & 0 & 1 & 1.610 & 0.246 & 1 & 1.640 \\
\hline & $2 \mathrm{pu}-1 \mathrm{pr}$ Conf & 0 & 1 & 1.269 & 0.194 & 1 & 1.281 \\
\hline 50 & $2 \mathrm{pu}-1 \mathrm{pr}$ & 8.240 & 0.745 & 1.644 & 0.247 & 0.780 & 1.684 \\
\hline & $2 \mathrm{pu}-1 \mathrm{pr}$ Conf & 8.240 & 0.622 & 1.301 & 0.195 & 0.652 & 1.320 \\
\hline 100 & $2 \mathrm{pu}-1 \mathrm{pr}$ & 16.449 & 0.301 & 1.794 & 0.249 & 0.386 & 1.893 \\
\hline & $2 \mathrm{pu}-1 \mathrm{pr}$ Conf & 16.445 & 0.143 & 1.476 & 0.197 & 0.189 & 1.531 \\
\hline 150 & $2 \mathrm{pu}-1 \mathrm{pr}$ & 24.527 & 0.064 & 2.693 & 0.271 & 0.161 & 3.127 \\
\hline & $2 \mathrm{pu}-1 \mathrm{pr}$ Conf & 24.248 & 0.012 & 3.097 & 0.218 & 0.043 & 2.952 \\
\hline 200 & $2 \mathrm{pu}-1 \mathrm{pr}$ & 30.398 & 0.023 & 3.470 & 0.316 & 0.098 & 3.400 \\
\hline & $2 \mathrm{pu}-1 \mathrm{pr}$ Conf & 30.586 & 0.005 & 2.531 & 0.247 & 0.022 & 2.732 \\
\hline
\end{tabular}


Table 1. Continued

\begin{tabular}{|c|c|c|c|c|c|c|c|}
\hline $\begin{array}{c}\text { Depth } \\
(\mu \mathrm{m})\end{array}$ & Ex. Mode & $\begin{array}{l}\text { Focal Shift } \\
\qquad(\mu \mathrm{m})\end{array}$ & Maximum N.I. & $\begin{array}{l}\text { Axial-FWHM } \\
(\mu \mathrm{m})\end{array}$ & $\begin{array}{c}\text { Radial-FWHM } \\
(\mu \mathrm{m})\end{array}$ & $\begin{array}{l}\text { Normalized } \\
\text { Signal Level }\end{array}$ & $\begin{array}{c}\text { Axial-P-FWHM } \\
(\mu \mathrm{m})\end{array}$ \\
\hline \multicolumn{8}{|c|}{$2 \mathrm{pu}-2 \mathrm{pr} ; \lambda_{\text {pump }}, 700 \mathrm{~nm} ; \lambda_{\text {probe }}, 700 \mathrm{~nm} ; \lambda_{\text {confocal }}, 450 \mathrm{~nm}$} \\
\hline \multirow[t]{2}{*}{0} & $2 \mathrm{pu}-2 \mathrm{pr}$ & 0 & 1 & 1.606 & 0.246 & 1 & 1.612 \\
\hline & $2 \mathrm{pu}-2 \mathrm{pr}$ Conf & 0 & 1 & 1.274 & 0.195 & 1 & 1.276 \\
\hline \multirow[t]{2}{*}{50} & $2 \mathrm{pu}-2 \mathrm{pr}$ & 8.231 & 0.743 & 1.631 & 0.247 & 0.760 & 1.636 \\
\hline & $2 \mathrm{pu}-2 \mathrm{pr}$ Conf & 8.231 & 0.620 & 1.298 & 0.195 & 0.643 & 1.306 \\
\hline \multirow[t]{2}{*}{100} & $2 \mathrm{pu}-2 \mathrm{pr}$ & 16.455 & 0.300 & 1.708 & 0.248 & 0.336 & 1.731 \\
\hline & $2 \mathrm{pu}-2 \mathrm{pr}$ Conf & 16.446 & 0.143 & 1.420 & 0.197 & 0.172 & 1.448 \\
\hline \multirow[t]{2}{*}{150} & $2 \mathrm{pu}-2 \mathrm{pr}$ & 24.645 & 0.063 & 1.948 & 0.251 & 0.097 & 2.054 \\
\hline & $2 \mathrm{pu}-2 \mathrm{pr}$ Conf & 24.486 & 0.012 & 2.067 & 0.204 & 0.025 & 2.086 \\
\hline \multirow[t]{2}{*}{200} & $2 \mathrm{pu}-2 \mathrm{pr}$ & 29.829 & 0.007 & 5.156 & 0.331 & 0.036 & 4.374 \\
\hline & $2 \mathrm{pu}-2 \mathrm{pr}$ Conf & 30.360 & 0.002 & 3.690 & 0.261 & 0.008 & 3.085 \\
\hline
\end{tabular}

${ }^{a}$ N.A., 0.75; N.I., normalized intensity; Axial-P-FWHM, axial plane FWHM; Conf, confocal.

pump-probe image formation for all combinations of pump-probe wavelengths for one- and two-photon procedures. The wavelength combinations (pump-probe) include $350-350,350-500,700-350,700-500$, and $700-700 \mathrm{~nm}$, and representative PSF plots are graphically illustrated in Figs. 3 and 4 . The complete results are tabulated in Table 1 . For comparison, the two-photon results have also been computed, and the results are shown in the same figures and in Table 1.

The representative axial and radial profiles of the PSFs are plotted in Figs. 3 and 4, respectively, and at depths of 0,50 , and $100 \mu \mathrm{m}$. From the results of our PSF calculations we derived four parameters that characterize the PSFs: focal shift, relative peak signal strength (to the surface), and the FWHMs of the axial and the radial PSFs. These numerical results are tabulated in Table 1. An examination of Figs. 3 and 4 and Table 1 yields several conclusions. First, the focal shift at each imaging depth is approximately a linear function of imaging depth and remains relatively unaffected by the wavelengths chosen for implementing the various modes of pump-probe microscopy. At imaging depths of 50, 100, 150, and $200 \mu \mathrm{m}$ the focal shift for all pump-probe modes and for two-photon microscopy is approximately 8, 16, 23, and $30 \mu \mathrm{m}$, respectively. These values represent an approximate shift of $15 \%$ of the focus from the geometrically unaltered focus. The second observation is the decrease in peak intensity and the increase in the widths of the PSFs along both the radial and the axial axes with increasing imaging depth. Comparatively, the depth-dependent PSF degradation is more significant along the axial axis. For example, consider the effects of spherical aberration in the one-photon pump, one-photon probe (1pu-1pr) case for which the pump and probe wavelengths are both $350 \mathrm{~nm}$. Table 1 lists the effects on the PSF at the surface and at depths ranging to $200 \mu \mathrm{m}$. Without confocal detection, the axial FWHM increased from 1.129 to $1.950 \mu \mathrm{m}$ (a $72.72 \%$ increase), whereas the radial FWHM increased from 0.174 to $0.231 \mu \mathrm{m}$ (a $32.76 \%$ increase). However, the normalized peak intensity decreased in a more dramatic fashion, from 1 to 0.039 . Note that, when the probe wavelength changes from 350 to $500 \mathrm{~nm}$ (stimulated emission), a different effect is observed. In the absence of a confocal detection pinhole, the FWHM of the axial PSF decreased from 1.300 to $2.717 \mu \mathrm{m}$ (a 109\% increase) from the surface to a depth of $200 \mu \mathrm{m}$. The radial FWHM, however, increased only from 0.199 to $0.263 \mu \mathrm{m}$ (a $32.16 \%$ increase) over the same depths. The maxima of the normalized intensity decreased to 0.047 of the surface peak value. These results indicate that pump-probe spherical aberration has the effect of decreasing the signal strength and redistributing the pump-probe signal into a larger volume along the axial axis. The effects are more significant with larger differences between the pump and the probe wavelengths. The normalized peak intensity of the PSF is dramatically affected by the choice of wavelengths used in implementing the different modes of signal generation. For example, at an imaging depth of $100 \mu \mathrm{m}$ the relative peak intensity increased from 0.085 for $1 \mathrm{pu}-1 \mathrm{pr}(350-350 \mathrm{~nm})$ to 0.300 for $2 \mathrm{pu}-2 \mathrm{pr}$ $(700-700 \mathrm{~nm})$; this value is further increased to 0.548 for two-photon (700-nm) excitation.

Finally, the addition of a confocal pinhole (at $450 \mathrm{~nm}$ ) has the tendency to decrease the peak PSF intensity and decrease the FWHMs of the PSFs. At an imaging depth of $100 \mu \mathrm{m}$ and for $2 \mathrm{pu}-2 \mathrm{pr}$ (700-700-nm) excitation, a confocal pinhole reduced the relative peak intensity from 0.300 to 0.143 (a decrease of 52.3\%), whereas the axial PSF decreased from 1.708 to $1.420 \mu \mathrm{m}$ (a decrease of $16.9 \%$ ) and the radial PSF decreased from 0.248 to $0.197 \mu \mathrm{m}$ a decrease of $20.6 \%$ ). In fact, from Table 1 one can see that, at large imaging depths, the loss of signal peak intensity consistently exceeds the relative gain in axial image resolution.

Note that in some cases in which the PSFs become highly aberrated, secondary peaks start to emerge and their amplitudes are comparable to the main intensity peaks. In such cases the identification of the FWHMs is not so easily achieved (see the curves for $100 \mu \mathrm{m}$ in Fig. 3). In those cases the axial FWHMs are computed by use of the Rayleigh criterion. Specifically, if the intensity minimum between the primary and secondary peaks is less than 0.5 of the 

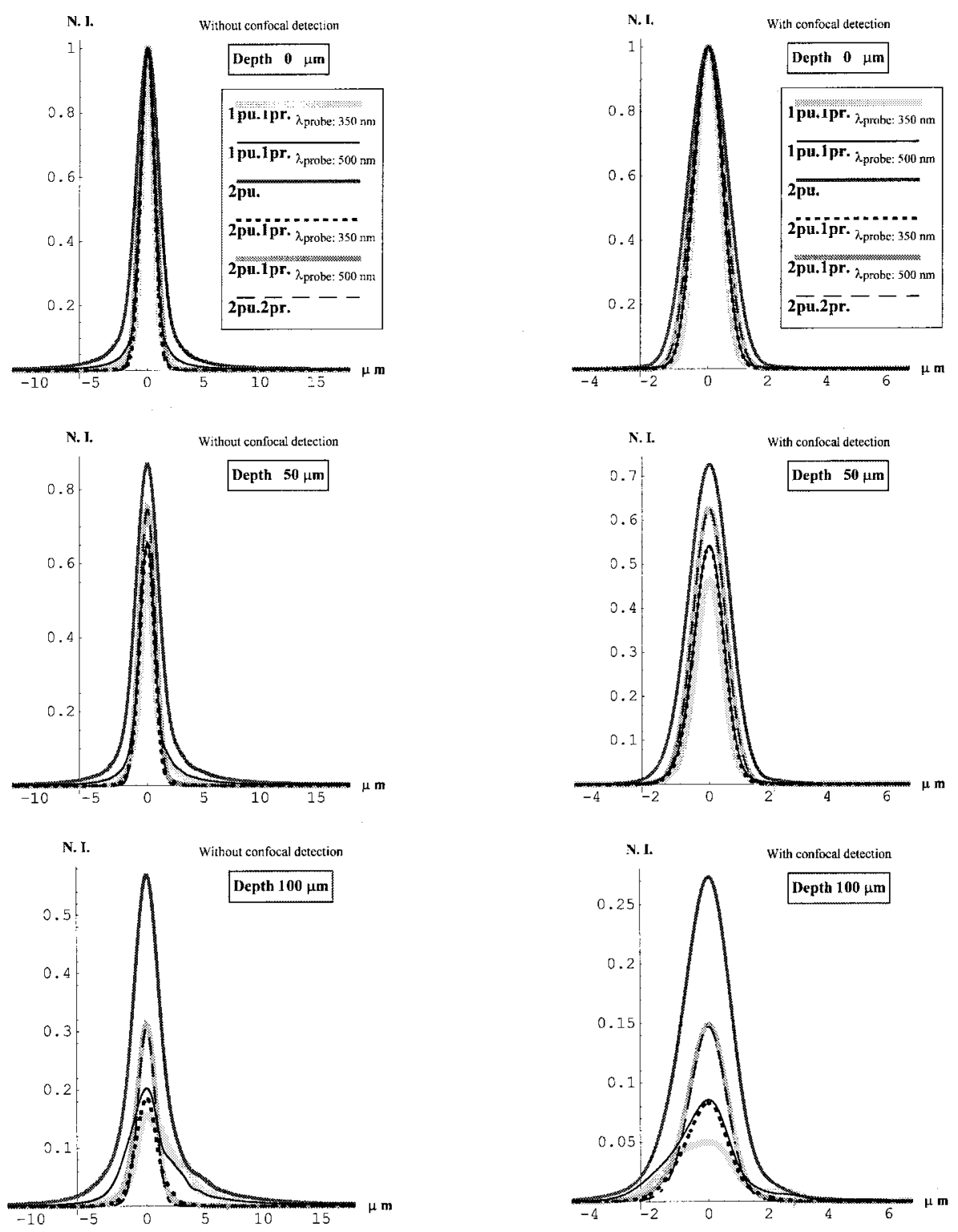

Fig. 5. Plane response of pump-probe and two-photon microscopy under spherical aberration.

primary peak, the width of the primary peak is determined to be the FWHM. Otherwise, the two peaks are treated as one peak and the PSF FWHM is computed as that of the combined peak.

In addition to PSF analysis, we also performed computations of the signal plane response and the total signal strength for several pump-probe configurations; representative plots are illustrated in Figs. 5 and 6 , respectively. The results are also tabulated in Table 1 along with the two-photon results. For example, consider the 1pu-1pr (350-350-nm) case without confocal detection. From 0 to $200 \mu \mathrm{m}$ the FWHM of the planar response increased from 1.193 to $3.517 \mu \mathrm{m}$ (a $194.8 \%$ increase) but the total signal level decreased only from $68.3 \%$ to 0.317 of the surface value. This finding further supports the fact that spherical aberration tends to redistribute the focal energy along the axial axis. With the addition of a confocal aperture, the FWHM of the axial planar response increased from 1.004 to $2.443 \mu \mathrm{m}$ (a 143.3\% increase) while the signal level decreased from the normalized value of 1 at the surface to 0.044 (a 95.6\% decrease). Therefore the redistribution of the pump-probe signal along the axial axis owing to spherical aberration results in decreased planar response degradation and a loss of total signal strength.

\section{Conclusions}

We have investigated the effects of refractiveindex-induced spherical aberration in pump-probe image formation for focusing through an oilwater interface [one-pump-one-probe (1pu-1pr), two-pump-one-probe (2pu-1pr), and two-pump-twoprobe (2pu-2pr) configurations]. As multiple wave lengths $(350,500$, and $700 \mathrm{~nm})$ can be used for pumpprobe bioimaging, it is conceivable that objective 
Without confocal detection
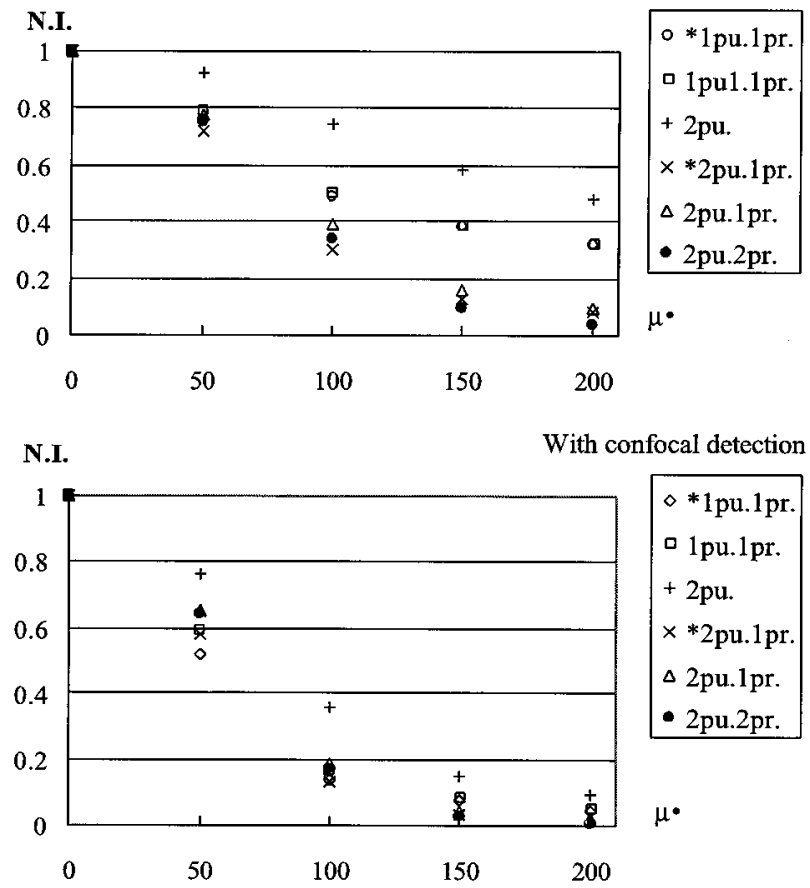

Fig. 6. Depth-dependent integrated signal strength of pumpprobe and two-photon microscopy under spherical aberration: *1pu.1pr., excitation with probe-beam wavelength $350 \mathrm{~nm}$; 1 pu.1pr., excitation with probe beam wavelength $500 \mathrm{~nm}$; *2pu.1pr., excitation with probe beam wavelength $350 \mathrm{~nm}$; 2 pu.1pr., excitation with probe beam wavelength $500 \mathrm{~nm}$. N.I., normalized intensity.

chromatic aberration can also be an important factor in determining the efficiency of pump-probe microscopy. This effect will be determined by the degree of chromatic aberration correction and is expected to be objective dependent. In practice, one should use the objectives with the highest degree of chromatic aberration in pump-probe imaging applications.

For spherical aberration we found that, whereas the focal shifts and the FWHMs of the radial PSFs are relatively unaffected by the different modes of pump-probe implementation, the relative peak intensity of signal and axial PSF widths can be drastically compromised. Analysis of the axial PSF, the planar response function, and the total signal strength show that in addition to causing signal loss, spherical aberration redistributes the pump-probe signal along the axial axis. The use of a confocal pinhole has the general tendency of reducing the peak intensity while improving both radial and axial PSFs. Furthermore, confocal detection reduces the FWHMs of both the axial planar response and the total signal strength. Our results show that, when one is applying pump-probe microscopy in bioimaging applications in which a mismatch of refractive indices occurs, a confocal pinhole should be used if the loss in signal strength at depth is not of concern; image resolution would improve. However, if one's goal is to acquire the maximum signal from pumpprobe imaging, the pinhole should not be used.

We acknowledge the support of grants NSC 912112-M-002 and NSC 92-2112-M-002-018 from the National Science Council, Taiwan, for this research. Peter T. Fwu and Po-Hsiang Wang made equal contributions to this paper.

\section{References}

1. W. Denk, J. H. Strickler, and W. W. Webb, "2-Photon laser scanning fluorescence microscopy," Science 248, 73-76 (1990).

2. P. T. C. So, C. Y. Dong, B. R. Masters, and K. M. Berland, "Two-photon excitation fluorescence microscopy," Annu. Rev. Biomed. Eng. 2, 399-429 (2000).

3. C. Y. Dong, P. T. C. So, T. French, and E. Gratton, "Fluorescence lifetime imaging by asynchronous pump-probe microscopy," Biophys. J. 69, 2234-2242 (1995).

4. C. Buehler, C. Y. Dong, P. T. C. So, T. French, and E. Gratton, "Time-resolved polarization imaging by pump-probe (stimulated emission) fluorescence microscopy," Biophys. J. 79, 536549 (2000).

5. C. Y. Dong, C. Buehler, T. C. So, T. French, and E. Gratton, "Implementation of intensity-modulated laser diodes in timeresolved, pump-probe fluorescence microscopy," Appl. Opt. 40, 1109-1115 (2001).

6. P. A. Elzinga, R. J. Kneisler, F. E. Lytle, G. B. King, and N. M. Laurendeau, "Pump/probe method for fast analysis of visible spectral signatures utilizing asynchronous optical sampling," Appl. Opt. 26, 4303-4309 (1987).

7. P. A. Elzinga, F. E. Lytle, Y. Jian, G. B. King, and N. M. Laurendeau, "Pump/probe spectroscopy by asynchronous opotical sampling," Appl. Spectrosc. 41, 2-4 (1987).

8. M. J. Booth, M. A. A. Neil, and T. Wilson, "Aberration correction for confocal imaging in refractive-index-mismatched media," J. Microsc. Oxford 192, 90-98 (1998).

9. M. J. Booth and T. Wilson, "Strategies for the compensation of specimen-induced spherical aberration in confocal microscopy of skin," J. Microsc. Oxford 200, 68-74 (2000).

10. M. J. Booth and T. Wilson, "Strategies for the compensation of specimen-induced spherical aberration in confocal microscopy of skin (vol 200, pg 68, 2000)," J. Microsc. Oxford 201, 416-416 (2001). 